\title{
Civilisations
}

Revue internationale d'anthropologie et de sciences

humaines

$65 \mid 2016$

Figures du malentendu

\section{La justification, l'explicitation et la dissociation comme remèdes au malentendu dans les discussions argumentatives}

\section{Anca Gâță}

\section{(2) OpenEdition}

12 Journals

Édition électronique

URL : http://journals.openedition.org/civilisations/4038

DOI : 10.4000/civilisations.4038

ISSN : 2032-0442

Éditeur

Institut de sociologie de l'Université Libre de Bruxelles

Édition imprimée

Date de publication : 19 décembre 2016

Pagination : $39-60$

ISBN : 2-9602017-0-3

ISSN : 0009-8140

Référence électronique

Anca Gâță, "La justification, l'explicitation et la dissociation comme remèdes au malentendu dans les discussions argumentatives », Civilisations [En ligne], 65 | 2016, mis en ligne le 19 décembre 2019, consulté le 15 janvier 2021. URL : http://journals.openedition.org/civilisations/4038 ; DOI : https:// doi.org/10.4000/civilisations.4038 


\title{
La justification, l'explicitation et la dissociation comme remèdes au malentendu dans les discussions argumentatives
}

\author{
Anca GÂT̆̌
}

\begin{abstract}
Résumé : Dans cet article, la notion de malentendu est précisée à travers des définitions de dictionnaires de la langue française des $19^{e}$ et $20^{e}$ siècles. Dans le cadre de la théorie actionnelle du langage, nous tentons de répondre à des questions concernant l'identification des actes de langage que les locuteurs peuvent qualifier de malentendus, mais aussi du gain interlocutif ou pragmatique issu de la qualification d'un incident comme un malentendu. L'étude donne des exemples de situations discursives où un incident, discursif ou non, est qualifié par les locuteurs de malentendu et elle identifie des (complexes d') actes discursifs ayant pour but de remédier à "quelque chose " qui a été qualifié ou présenté comme un malentendu. Des extraits de prises de parole dans les réunions et les communiqués des institutions européennes illustrent l'analyse. Le concept de dissociation emprunté à la rhétorique et à la théorie de l'argumentation permet de montrer que les locuteurs essaient de remédier à un malentendu par des précisions qui remettent en discussion les prémisses d'un échange en proposant des réinterprétations notionnelles des termes qui pourraient être à la base d'un incident considéré comme un malentendu. Or, certains incidents, bien que présentés comme des malentendus, et donc comme des erreurs réparables ou sans grande importance, sont en fait irréversibles.
\end{abstract}

Mots-clés : argumentation, dissociation (argumentative), malentendu, rhétorique, stratégies persuasives.

\begin{abstract}
The notion of misunderstanding is approached in this article through definitions given in French dictionaries of the $19^{\text {th }}$ and $20^{\text {th }}$ centuries. In an action-oriented theory of language, we identify speech acts acknowledged by the speakers as misunderstandings, and discuss how speakers might have a locutionary or pragmatic gain in interpreting a speech act as a misunderstanding. The study provides examples of discursive situations in which an incident, discursive or not, is labelled as a misunderstanding by the speakers and it identifies (complexes of) speech acts supposed to resolve the misunderstanding. The analysis is illustrated with excerpts from discussions in the meetings and press releases of the European Union institutions. The concept of dissociation borrowed from rhetoric and argumentation theory is used to show that speakers try to resolve misunderstanding by specifying the starting points (or premises) of the discussion, and propose notional reinterpretations of a term. Some of these situations, although presented as misunderstandings and hence as errors of lesser importance for which one could find solutions, are in fact impossible to rectify.
\end{abstract}

Keywords: argumentation, (argumentative) dissociation, misunderstanding, rhetoric, persuasive strategies. 


\section{Introduction}

Cet article $^{1}$ propose une approche linguistique de la notion de « malentendu ». Je tente d'en proposer une typologie simple, qui puisse servir de base à des études ultérieures et plus contextualisées. C'est pourquoi je m'appuie sur des sources diverses et relativement hétéroclites. Je m'intéresserai en effet non seulement à des énoncés oraux, mais aussi à des énoncés oraux présentés sous forme écrite, ou encore à des descriptions de situations trouvées dans la littérature de fiction. Dans cet article, j'entends répondre aux questions suivantes :

1) Qu'est-ce que les locuteurs ordinaires présentent comme, ou qualifient du terme de malentendu?

2) Quels types d'actes de langage sont effectués dans une situation discursive où les locuteurs font référence, implicitement ou (semi) explicitement à un malentendu ?

3) Quel est le gain interlocutif ou pragmatique tiré de la présentation d'un événement en terme de malentendu?

Je commencerai par présenter des définitions lexicographiques de la notion de « malentendu » inventoriées dans quelques dictionnaires de la langue française des $19^{\mathrm{e}}$ et $20^{\mathrm{e}}$ siècles. Dans un deuxième temps, je proposerai une typologie simple des situations que l'on peut qualifier de malentendu. La perspective de la théorie des actes de langage sera ensuite appliquée à la description de certains énoncés qui font référence à un malentendu, de manière implicite ou explicite. Enfin, dans la section finale, je discuterai des situations discursives où un locuteur présente « quelque chose » comme un malentendu, de manière explicite (en le désignant comme tel) ou implicite - ces deux dernières parties s'attacheront à montrer comment les locuteurs proposent de remédier à ce qu'ils présentent comme malentendu. Nous verrons également que certaines situations ne permettent pas de remédier aux dégâts causés par un malentendu. Dans tous ces cas, l'article s'intéressera au potentiel rhétorique des énoncés portant sur une situation présentée comme relevant d'un malentendu.

Commençons par une idée simple. Je suivrai Marcelo Dascal (1999 : 754) lorsqu'il affirme que le malentendu « est typiquement un phénomène communicatif involontaire reposant plutôt sur une incorrection que sur une conduite non éthique, et qui s'inscrit dans un processus de réception tant aux niveaux sémantique que pragmatique $»^{2}$.

Lors d'une enquête informelle relatée dans son article « No conversation without misrepresentation » (1993), le linguiste américain Bruce Fraser a demandé à une série de personnes si elles pensaient que, dans leurs conversations quotidiennes, il était courant de présenter les choses de manière erronée. Les réponses étaient négatives : les

1 Il se fonde sur une étude réalisée dans le cadre du programme annuel 2016 du Centre de recherches Théorie et Pratique du Discours de l'Université Dunărea de Jos de Galaţi, Roumanie, et du projet Soutien aux formations de licence en français, sous la coordination de Gabriela Scripnic, financé par l'Agence Universitaire de la Francophonie (BECO - Bucarest). 
personnes interrogées estimaient que les gens ne font pas ce type d'erreur, exception faite des politiciens et des administrateurs de l'université. En dépit de ces réponses, fondées sur des intuitions plus ou moins correctes, Fraser considère que les locuteurs ont souvent tendance à présenter les choses de manière erronée (misrepresent), que ce soit volontairement ou non, ce qui revient à transmettre des informations fausses (1993 : 143). La représentation incorrecte involontaire peut avoir comme origine une faute commise par le locuteur en produisant un énoncé, et cette représentation peut engendrer un malentendu. Si Fraser pense, comme l'indique le titre de son article, qu' " il n'y a pas de conversation sans représentation fautive », c'est que, d'une part, personne ne peut vérifier toutes les informations transmises lors d'une conversation afin d'établir leur véracité et que, d'autre part, la " vérité » d'un énoncé peut changer dans le temps. Ce qui était vrai un jour ne le sera peut-être plus le lendemain. Frazer dégage une typologie des actes discursifs de représentation fautive ou incorrecte de la réalité, dont je donne un aperçu schématique ci-dessous :

a) des énoncés/actes de langage produits avec l'intention de tromper le destinataire. Le locuteur ment ;

b) des énoncés/actes de langage produits sans l'intention de tromper l'autre. Le locuteur plaisante ;

c) le locuteur produit une représentation incorrecte de la réalité sans le vouloir et sans intention de tromper l'autre. Le locuteur se trompe et peut dès lors tromper l'autre.

On voit donc que la représentation incorrecte de la réalité peut être volontaire ou involontaire. C'est le dernier type qui nous intéresse dans la question du malentendu. (cf. Fraser, $1993:$ 144-146)

\section{A propos de la notion de « malentendu »}

Erreur, égarement, bévue, méprise, quiproquo, malentendu, mécompte, illusion, prestige $^{3}$, préoccupation, prévention, préjugé - telle est la liste des mots que Pierre Benjamin Lafaye réunit en 1837 dans l'article « erreur » qu'il rédige comme co-auteur de l'Encyclopédie des gens du monde. Il définit l'erreur comme l' « état dans lequel notre esprit, voyant les choses autrement qu'elles ne sont, porte sur elles un faux jugement, ou bien ce faux jugement lui-même » (Lafaye, 1837 : 735). Le malentendu est ainsi rangé avec la bévue, la méprise, le quiproquo, le mécompte, parmi les erreurs « passagères, momentanées » que l'on peut commettre, « qui dépendent de causes accidentelles, comme une distraction, une inadvertance, une surprise », de " la légèreté de l'esprit » ou du « mauvais emploi de nos facultés intellectuelles » (ibid., 736), engendrées par un esprit « qui se trompe de sang-froid, avec ingénuité, sans s'en douter, sans le vouloir, et dans un cas bien particulier où il lui eût été facile de juger sainement » (ibid., 737).

La notion de malentendu a donné lieu à des définitions plus ou moins élaborées dans les dictionnaires de la langue française ou dans d'autres ouvrages s'efforçant de donner une description notionnelle aussi détaillée que possible de divers termes. Les définitions ci-dessous correspondent aux entrées « malentendu » de plusieurs

3 Le mot « prestige » utilisé par Lafaye a le sens d' « illusion », « charme », « attrait », se rapportant à cette dimension de fascination, qui intervient en effet dans la constitution de certains malentendus. 
dictionnaires et peuvent donner une idée de la représentation que l'on s'est faite en France de cette notion au cours des siècles :

(1) Paroles prises dans un autre sens qu'elles n'ont été dites.

(1a) Ils ne s'expliquèrent pas bien et le malentendu causa une grande contestation. (Dictionnaire de l'Académie française (ci-après $D A F, 1814$ )

(2) Actions mal interprétées et qui produisent quelque division. $(D A F, 1814)$

3) Erreur, méprise.

(3a) Il y a du malentendu dans cette affaire.

(3b) Un malentendu lui a fait perdre un procès. $(D A F, 1814)$

(4) Parole, discours mal interprété(e).

(4a) Ils se brouillèrent pour un malentendu.

(4b) Il fut condamné par un malentendu. (Laveaux, 1820)

(5) Méprise qui empêche de s'entendre, d'être d'accord.

(5a) Il y a des malentendus entre nous et les Anglois.

(5b) N'y a-t-il pas entre nous quelque malentendu qui vient peut-être de ce qu'il ne sait pas assez bien le français? (Darmesteter et Hatzfeld, 1890)

(6) Divergence d'interprétation sur la signification de propos ou d'actes entraînant un désaccord.

(6a) Quel malentendu incroyable! Et moi qui avais compris le contraire! Mais enfin, comment ne vous êtes-vous pas expliquée? (Montherlant, Pitié pour les femmes, 1936, p. 1113)

(6b) ... il y a, fatalement, à la base de tout amour passionné, un malentendu, une illusion généreuse, une erreur de jugement : une conception fausse qu'on s'est faite l'un de l'autre... (Martin du Gard, Les Thibault, L'Epilogue, 1940, p. 839) (TLF)

(7) Désaccord entraîné par une telle divergence.

(7a) C'est la tricherie d'amour qui fera naître les malentendus les plus graves, les plus durables, et les rancunes les plus véhémentes. (Jankélévitch, Le Je-ne-saisquoi et le presque-rien, 1957, p. 163)

(7b) Le désaccord n'avait fait que grandir, aggravé par un de ces singuliers malentendus de la chair qui glacent les plus ardents : il adorait sa femme, elle était d'une sensualité de blonde gourmande, et déjà ils couchaient à part, mal à l'aise, tout de suite blessés. (Zola, Germinal, 1885, p. 1305) (TLF) 
On voit donc que le malentendu est perçu comme un incident de communication ou d'interprétation, dont l'origine peut être une action mal interprétée ou un acte de communication raté. Ce qui revient à dire que les actes et les faits d'un individu sont mal saisis, ou que ses dires sont interprétés autrement qu'ils ne devaient l'être.

Selon ces définitions, le malentendu peut incomber à l'auteur des dires ou des faits, à l'interprétant, ou bien aux deux parties - ou acteurs - de l'interaction. Les définitions mettent en évidence la principale conséquence d'un malentendu : la différence d'opinion ou le désaccord. Le désaccord issu d'un malentendu peut être de nature idéelle - les « actants » du malentendu ont des représentations différentes de la même situation ou bien de nature actionnelle - les « actants » du malentendu peuvent agir de manière divergente ou contraire quand des actions consensuelles s'imposaient (voir à cet effet l'exemple de la situation présentée dans la dernière section de l'article).

L'exemple (6a) montre qu'un malentendu peut avoir des dimensions importantes ; il peut être qualifié d'incroyable pour parler de son ampleur, de sa portée, du fait que les opinions des deux parties en interaction sont contraires, et les exemples (1a), (3b), (4a), (4b), (7a) montrent qu'un malentendu peut avoir des conséquences graves, insurmontables, irrémédiables, définitives.

On observe aussi que le terme malentendu peut être utilisé dans un sens figuré, métaphorique, synonyme de «mésentente», « dissension », «brouille», « dissentiment», comme dans les contextes des exemples (7a) et (7b), mais ce n'est pas de ce type d'emploi dont il sera question ici.

Le Trésor de la langue française (ci-après $T L F$ ) fournit également une série de collocations et d'expressions usuelles comportant le terme malentendu : dissiper un malentendu ; (pour) éviter tout malentendu ; malentendu qui sépare deux personnes, qui se glisse entre deux personnes; c'est un malentendu; il y a (un) malentendu (entre deux personnes). Ces expressions mettent en évidence les aspects suivants :

1) C'est un malentendu peut décrire des propos dans le cadre d'un échange conversationnel, ou des actions interprétées de manière fautive ;

2) Il y a (eu) (un) malentendu peut qualifier l'acte d'interprétation de certains propos ou actions quand le destinataire du message ou un tiers a compris les faits ou les dires de quelqu'un de manière fautive. A la différence de l'expression précédente, cette expression permet de souligner de manière explicite la divergence survenue entre deux sujets ;

3) [le] malentendu qui sépare deux personnes renvoie au même type de divergence en insistant sur les positions différentes qu'occuperont dans l'interaction (linguistique ou non) les deux personnes suite au malentendu ;

4) [un] malentendu qui se glisse entre deux personnes fait référence au caractère insidieux du malentendu;

5) (pour) éviter tout malentendu suggère que les sujets sont intéressés par des actes et des propos suffisamment bien précisés et délimités pour que le malentendu soit évité ;

6) dissiper un malentendu montre qu'un malentendu n'est pas une situation insurmontable, que l'éliminer ne requiert pas des efforts importants et, par une exploitation des relations synonymiques du verbe dissiper, qu'un malentendu peut être aussi écarté, balayé, éliminé, éclairci, qu'on peut y mettre fin, le faire disparaître.

Laveaux (1826 : 187), redéfinit le malentendu, comme suit : 
Le malentendu n'est pas, comme on l'a dit, une erreur qui vient de ce qu'on a mal entendu ou mal compris quelque chose. L'erreur qui vient de ce qu'on a mal entendu ou mal compris un passage de Perse ou de Tacite n'est pas un malentendu. Le malentendu est une erreur qui résulte de ce qu'une personne a dit à une autre personne de faire une chose, dans un certain temps, à une certaine époque, de telle ou telle manière, et que celle-ci a mal entendu, mal compris ce qu'a dit la première; de manière qu'elle a fait une autre chose, ou qu'elle fait la chose dans un autre temps, ou d'une autre manière. Vous m'aviez promis de venir me prendre chez moi à midi, j'ai cru que vous aviez dit à une heure; c'est un malentendu. Le malentendu est une erreur qui a lieu entre les hommes dans le commerce de la vie. Il n'est point applicable à d'autres espèces d'erreurs. Une erreur de chronologie, une erreur de physique, ne sont pas des malentendus. (en italique dans le texte)

La citation ci-dessus attire notre attention sur la nature essentiellement énonciative et communicative du malentendu dans le sens où il est issu d'un acte de langage. Elle attribue explicitement la responsabilité du malentendu au récepteur. Le malentendu se rapproche de la notion de quiproquo, bien que celle-ci ne repose pas uniquement sur des actes de langage. Le même auteur présente le quiproquo de la manière suivante :

Le quiproquo est aussi une erreur qui a lieu dans le commerce de la vie. C'est la méprise d'une personne qui a donné, pris, ou fait, ou dit une chose pour une autre. On appelle précisément quiproquo d'apothicaire, la méprise d'un apothicaire qui délivre à une personne un remède préparé pour une autre; ou qui, dans la composition d'un médicament, emploie une drogue pour une autre. On le dit aussi par extension de toutes les fautes ou méprises qui se commettent en médecine, soit dans l'ordonnance, la préparation ou l'application des remèdes. (en italique dans le texte)

Cet auteur établit une distinction entre ce qu'il appelle malentendu, qui repose sur un dysfonctionnement communicatif - donc strictement linguistique -, et le quiproquo, qui repose sur tout acte pouvant susciter une méprise, y compris un acte communicatif. L'emploi courant de ce dernier terme étant assez restreint ou limité à certaines situations, il semble avoir été remplacé dans l'usage commun et courant par le terme malentendu, dont le sens, plus accessible au locuteur ordinaire, aurait été ainsi étendu pour intégrer aujourd'hui les deux significations fondamentales discutées ci-dessus. C'est pourquoi les analyses qui suivent reposeront tant sur le malentendu que sur ce que Laveaux aurait appelé le quiproquo.

\section{Essai de typologie}

Afin de pouvoir se représenter une situation comme un malentendu, il faut qu'un locuteur identifie une certaine inconséquence d'action ou un acte discursif incorrect comme la cause de la situation en question. En effet, le malentendu n'existe pas en dehors de la saisie d'un acte antérieur comme fautif ou incorrect. Présenter une situation - discursive ou non - comme un malentendu revient à utiliser des expressions prototypiques telles que : c'est un malentendu, ce n'est qu'un malentendu. Toutes sortes d'autres développements énonciatifs peuvent remplacer de telles expressions. Dans de tels cas, la situation n'est pas qualifiée de malentendu mais présentée comme telle de manière implicite. On peut dire, par exemple : non, je ne voulais pas dire ça, 
je voulais dire autre chose, tu n'as pas compris, je ne comprends pas alors ce que tu veux, mais je croyais que tu parlais d'autre chose, etc ${ }^{4}$. Quoi qu'il en soit, pour que l'on puisse recourir légitimement à la notion de « malentendu », il faut que la situation ait été présentée comme telle de manière implicite ou (semi-) explicite par l'un des interactants ou un tiers observateur. Comme les exemples qui suivent le montrent, ce principe sert de pierre de touche à la typologie que je propose.

Examinons la situation suivante :

Je l'ai vue traverser la rue, je croyais qu'elle m'avait vu, j'allais me diriger vers elle, mais elle entra rapidement dans la boutique - elle ne m'avait pas vu, je m'étais trompé.

Est-ce qu'on pourrait qualifier cette situation de malentendu? Est-ce que le locuteur pourrait dire ce fut/c'était un malentendu (de ma part) ? Il est difficile de considérer cette situation comme un malentendu et de la qualifier comme telle, car l'autre personne n'a pas fait d'action intentionnelle par rapport au locuteur ; elle n'était même pas au courant de la présence de ce dernier. Or, toutes les définitions reprises ci-dessus mentionnent au moins deux interactants. Il faut que le geste, l'action de l'un soit en quelque sorte destiné à l'autre ou aux autres. Dans le cas présenté il s'agit d'une erreur d'interprétation, pas d'un malentendu.

C'est ce dont il est question dans le passage de $A$ la recherche du temps perdu, où le narrateur décrit la situation d'un voyageur malade hébergé dans un hôtel, qui commet deux erreurs d'interprétation : la « raie de jour » qu'il aperçoit sous la porte n'annonce pas le matin comme il l'avait espéré, et les pas qu'il croyait être ceux d'un domestique se portant à son secours se révèlent être ceux d'un quidam vacant à d'autres occupations :

Bientôt minuit. C'est l'instant où le malade, qui a été obligé de partir en voyage et a dî coucher dans un hôtel inconnu, réveillé par une crise, se réjouit en apercevant sous la porte une raie de jour. Quel bonheur ! c'est déjà le matin ! Dans un moment les domestiques seront levés, il pourra sonner, on viendra lui porter secours. L'espérance d'être soulagé lui donne du courage pour souffrir. Justement il a cru entendre des pas; les pas se rapprochent, puis s'éloignent. Et la raie de jour qui était sous sa porte a disparu. C'est minuit; on vient d'éteindre le gaz ; le dernier domestique est parti et il faudra rester toute la nuit à souffrir sans remède. (Marcel Proust, Du côté de chez Swann) ${ }^{5}$ [mes italiques dans le texte, A.G.]

Dans cet exemple, le sujet qui perçoit et interprète la situation est en quelque sorte dédoublé : d'abord sujet et «patient », ou récepteur, de l'interprétation première, fautive, il est ensuite sujet et récepteur d'une deuxième interprétation correcte. C'est dans ce sens que l'on pourrait recourir au terme de malentendu, quoique la désignation la plus adéquate pour une telle situation soit l'« erreur d'interprétation ».

4 Il pourrait être intéressant d'inventorier de telles expressions ; les forums électroniques en constituent un réservoir inépuisable.

$5<$ alarecherchedutempsperdu.org $>$. 
A partir des distinctions notionnelles rappelées dans la section précédente entre malentendu et quiproquo et des fragments situationnels exposés ci-dessous, on peut identifier les classes de malentendu suivantes :

I. Il peut être issu de la mauvaise interprétation des actions d'un individu, comme dans la séquence décrivant une personne traversant la rue et saluant une autre.

I.a. Il n'est pas qualifié comme tel par le locuteur - la référence au malentendu est implicite.

I.b. Si le locuteur ajoute $C$ 'était un malentendu, la référence est explicite.

II. Il peut être issu de la mauvaise interprétation des propos d'un locuteur, comme dans le dialogue suivant :

$A$ : J'ai faim.

$B$ : Prends une chouquette.

$A$ : Mais tu sais que je suis au régime. Je vais m'acheter un yaourt.

$B:$ Ah, bon...

II.a. C'est un malentendu qui n'est pas qualifié comme tel par l'interlocuteur B ; la référence au malentendu demeure implicite. Le locuteur A n'avait pas produit son énoncé pour demander à $\mathrm{B}$ quelque chose à manger, mais pour lui faire connaître sa sensation de faim, son " état », et peut-être son intention de sortir s'acheter à manger. Il rectifie l'énoncé initial dont le contenu littéral avait une autre valeur d'interprétation en faisant connaître son souhait de s'acheter à manger, ce qui entraîne l'interlocuteur B à

II.b. répondre $A h$, bon.... S'il avait répondu $A h$, bon. C'était un malentendu, il aurait rendu la référence au malentendu explicite.

Ces exemples simples montrent qu'à la différence d'autres erreurs, un acte d'interprétation peut être qualifié de malentendu par l'emploi d'un énoncé du type c'est un malentendu. Cet énoncé fait référence à une différence, ou bien à une erreur d'interprétation de faits - qu'il s'agisse de « faits de discours », ou d'actes de discours -, mais les locuteurs peuvent être tout aussi conscients qu'ils ont mal interprété un fait ou des paroles en n'utilisant pas une qualification discursive de leur interprétation comme malentendu.

\section{Le malentendu saisi à travers la théorie des actes de langage}

Une possibilité d'étudier la notion de malentendu du point de vue linguistique est d'approcher les situations de malentendu par le biais de la théorie des actes de langage et de l'analyse du discours. La notion d'acte de langage implique de saisir la nature actionnelle du langage, de manière à penser tout énoncé produit par un locuteur comme un acte et non comme de simples paroles, dont seul compterait le contenu propositionnel.

La typologie des actes de langage proposée par Austin (1962) et reprise par Searle $(1969,1979)$ peut servir de point de départ à une approche de la notion de malentendu du point de vue énonciatif. L'acte illocutoire, constitutif de l'acte de langage global, souligne l'acte effectué en disant quelque chose. Par exemple, l'acte illocutoire correspondant à l'énoncé Je promets de t'aider est la promesse. Conformément à la taxinomie de Searle (1979), les actes illocutoires sont : les assertifs, les directifs, les promissifs, les expressifs et les déclarations. Un acte illocutoire appartenant à chacune 
des quatre premières catégories pourrait être à l'origine d'un malentendu. Les actes illocutoires de déclaration, ou déclarations (Searle, 1979 : 56), qui reposent sur une convention sociale ou institutionnelle et qui permettent de créer un nouvel état du monde - une nomination, une nouvelle relation sociale, civile -, ne semblent pas pouvoir se trouver à l'origine d'un malentendu, étant donné leur caractère conventionnel, l'emploi du discours écrit pour les mettre en place et leur fonctionnement souvent public. Il faut également tenir compte du fait que les quatre catégories d'actes mentionnées plus haut peuvent se produire en tant qu'actes illocutoires indirects, ce qui accroît la possibilité qu'ils soient source de malentendus. L'acte de langage indirect prend la forme d'un énoncé dont la force, ou valeur, illocutoire n'est pas explicite. Je vais t'aider peut, par exemple, fonctionner comme une promesse. L'énoncé Je suis toujours ici peut servir à accomplir un acte indirect de promesse, et dans ce cas il signifie « je promets que je t'attends ». Mais l'énoncé peut tout aussi bien ne pas être une promesse, transmettre un reproche et signifier « je travaille plus que toi » ou bien « je fais toujours mon travail tandis que tu ne fais rien à temps ». Le succès d'un acte de langage repose sur la sincérité du locuteur et sur la bonne volonté de l'interlocuteur, sur la coopération des interlocuteurs.

Le concept d'acte de langage rend sensible le fait que, dans toutes les instances de communication, un locuteur transmet un message constitué d'un contenu propositionnel et d'une force illocutoire. La force illocutoire de l'énoncé représente la valeur que revêt ce dernier dans la situation d'interlocution : tel énoncé sert à poser une question, tel autre à exprimer un état psychologique du locuteur, etc.

Le malentendu peut concerner la force illocutoire de l'énoncé, le contenu de l'énoncé, ou bien les deux. Je ne ferai pas ici la distinction entre discours oral et discours écrit, bien qu'elle soit nécessaire et peut-être éclairante pour la problématique du malentendu, car la façon dont on produit les énoncés à l'oral a un impact sur la manière dont on comprend leur contenu.

On peut reprendre l'exemple d'interaction présenté ci-dessus pour rendre explicites les notions abordées jusqu'ici. Le locuteur A produit l'énoncé J'ai faim avec une force illocutoire qui pourrait être interprétée comme assertive au premier niveau, c'est-à-dire comme un énoncé par lequel il donne une information concernant un état de fait. Mais la force illocutoire assertive n'est pas acceptable à elle seule, car l'énoncé ne fournit pas de véritable information sur le monde comme le font les assertifs. Ainsi pourraiton identifier une force illocutoire de deuxième niveau, expressive, qui présenterait l'énoncé comme chargé de communiquer l'état psychologique du locuteur A : il se plaint du malaise provoqué par la faim. L'interlocuteur B ne trouve pas d'intérêt à cette force expressive de l'énoncé, de sorte qu'il en dérive une force illocutoire directive à un troisième niveau, ce qui veut dire qu'il peut interpréter l'énoncé comme un ordre, une demande, une prière. De cette façon, il comprend l'énoncé en tant que requête et offre à manger au locuteur $\mathrm{A}$. Mais celui-ci repousse cette interprétation : son énoncé n'a pas la force illocutoire d'une demande - il ne demande pas quelque chose à manger. Le locuteur A réinstaure pour l'énoncé initial une force illocutoire expressive - il veut simplement dire son malaise, tout en ne voulant pas manger car il fait un régime - ou bien assertive - son énoncé devrait alors être compris comme une explication de son acte prochain de sortir, comme « Je vais sortir me chercher à manger (parce que j'ai faim) ». Le malentendu se situe au niveau de la force illocutoire en tant que composante 
de l'acte de langage et non au niveau du contenu propositionnel qui n'est pas le siège d'un malentendu.

\section{Qu'est-ce qu'on présente comme malentendu et comment ?}

J'ai suggéré que les locuteurs utilisent le terme malentendu pour désigner ou décrire un dysfonctionnement dans la communication ou dans l'interaction. Examinons les situations dans lesquelles un locuteur peut présenter, de manière implicite ou (semi-) implicite, une situation comme relevant d'un malentendu. Les quelques exemples suivants sont puisés dans des sténogrammes des réunions d'institutions européennes ou empruntés à des communiqués de presse présentant des interventions dans le cadre de ces mêmes institutions. Soulignons d'abord trois situations types :

a) Toutes les situations qualifiées de « malentendu » n'en sont pas ; elles peuvent relever d'ambiguïtés sémantiques ou syntaxiques, d'ironie, d'erreurs de jugement, etc.

b) De nombreuses situations peuvent relever du malentendu sans qu'un tiers ou bien les participants, les interactants, ne les désignent ou les qualifient explicitement comme telles. Pourtant, les destinataires de l'énoncé sont censés interpréter ces énoncés ou ces faits comme étant à l'origine d'un malentendu. Dans ce cas, sans recourir au terme de malentendu, le locuteur laissera entendre que, de son point de vue, « il y a malentendu ».

Dans l'extrait ci-dessous, emprunté à un sténogramme de séance du Parlement européen, madame Sinclaire, la locutrice, présente des critiques concernant la modalité de vote sur un rapport traitant de la transparence. Dans sa prise de parole, elle établit une distinction entre le vote qu'elle a effectué contre une procédure de vote et son opinion personnelle quant aux mesures de transparence qu'elle était prête à soutenir. Ce qui est en jeu c'est la procédure de vote par appel nominal (un vote qui n'est pas secret, mais public, par lequel la personne est appelée à présenter son vote pour ou contre ou son abstention de manière publique, tant pour les membres de l'assemblée que pour les électeurs de son pays) :

Nicole Sinclaire : - $(E N)^{6}[\ldots]$ Ma deuxième critique concerne un refus de me
laisser présenter une explication de vote au sujet du rapport Tremopoulos. Il
s'agissait d'un vote par appel nominal concernant la transparence, et j'ai voté
contre. A présent, on ne me donne pas l'occasion d'expliquer pourquoi j'ai voté
contre, mais mes électeurs verront que j'ai voté contre la transparence. Je veux
pouvoir expliquer pourquoi j'ai voté contre ce rapport. Permettez-moi, je vous
prie, de présenter une explication de mon vote à ce sujet. (15 juin 2010)

La locutrice évoque un malentendu potentiel, qu'elle ne qualifie d'ailleurs pas comme tel. Elle anticipe l'erreur que pourraient commettre ses concitoyens en prenant son vote pour un refus de la transparence. Elle précise que son vote n'était pas tourné contre la transparence en tant que telle, mais contre la procédure du vote par appel nominal, qui rendait public le vote alors qu'elle aurait voulu le garder secret. Elle dit

6 Les initiales placées au début du texte du sténogramme indiquent la langue dans laquelle le discours a été prononcé. Dans ce cas, le texte original était en anglais. La plupart des interventions sont disponibles dans les langues les plus importantes des organismes européens, la traduction étant fournie par l'organisme européen en question. 
que ses électeurs "verront qu'elle a voté contre », ce qui veut dire qu'en l'absence des explications qu'elle souhaite apporter, ils « comprendront » son vote de manière erronée. On peut accepter pour les besoins de cette analyse qu'elle parle de manière implicite de l'éventualité d'un malentendu dans le chef de ses électeurs qui ne seraient pas bien informés. Elle exige donc d'apporter des précisions et des clarifications, et elle le fait par le biais d'un acte de langage implicite de distinction entre le vote contre la transparence, dont elle pourrait être accusée, et le vote par appel nominal (concernant la transparence).

c) Des malentendus peuvent être signalés de manière semi-explicite par des expressions du type : pour m'expliquer; pour vous faire comprendre; juste pour mieux comprendre; ce que vous êtes en train de nous dire, c'est que..., comme dans l'extrait ci-dessous. Dans un dialogue, de telles expressions laissent entendre que des propos antérieurs peuvent avoir été mal compris ou sont déficitaires du point de vue de l'expression. Les deux premières expressions ci-dessus donnent à entendre que le locuteur voudrait reformuler ses propos, comme c'était aussi le cas pour madame Sinclaire, dans le paragraphe précédent. Les deux expressions suivantes pourraient apparaître dans des situations où c'est l'interlocuteur qui propose une reformulation et demande au premier locuteur une confirmation concernant l'adéquation de cette dernière. L'exemple suivant le montre :

Stavros Lambrinidis - (EN) Monsieur le Président, juste pour mieux comprendre, ce que vous êtes en train de nous dire, c'est que lors de l'élection d'un viceprésident, il est impossible de ne pas obtenir de majorité qualifiée, vu que s'il est seulement possible de voter pour ou de s'abstenir et que les abstentions ne sont pas comptabilisées dans le total des votes, alors, par définition, on obtient forcément une majorité qualifiée. Est-ce bien ce que vous êtes en train de nous dire? (15 juin 2010) [mes italiques dans le texte, A.G.]

Ces cas de figure ne sont pas discutés dans l'étude présente, mais ils sont extrêmement intéressants du point de vue linguistique. Il est plus difficile d'identifier et d'inventorier des situations communicatives illustrant les points a) et b) car il n'y a pas de marqueurs linguistiques et l'analyste devrait parcourir un nombre immense de textes ou de transcriptions de discours oraux afin de relever des malentendus qui ne sont pas désignés comme tels.

\section{Remédier au malentendu : mouvements rhétoriques de justification d'explicitation, de dissociation}

On peut remédier aux divers malentendus apparaissant dans la communication ou dans les conduites communicatives qualifiées comme telles par des actes communicatifs ultérieurs. Du moins, on constate que les locuteurs ressentent le besoin de reformuler leurs dires ou les dires des autres qu'ils sont censés éclaircir, qu'ils proposent des justifications et des explicitations afin de dissiper le malentendu et éviter ou atténuer un désaccord qui peut apparaître dans des situations argumentatives. Dans d'autres cas, les locuteurs qualifient de malentendu une situation qui ne dépend pas d'eux, et tentent de l'expliquer en montrant quelles en sont les bases ou les causes.

L'emploi du terme malentendu peut, par exemple, renforcer un acte expressif d'excuse en indiquant la cause d'un acte raté comme appartenant à un domaine délimité 
de la réalité objective, la réalité langagière. Ainsi, la justification d'un acte raté par un « malentendu » peut rendre à nouveau crédible un locuteur décrédibilisé par un acte de communication raté. C'est le cas de l'allocution du président en exercice du Conseil de l'Union Européenne, Ivan Lewis, qui arrive en retard à une réunion du Parlement européen :

Ivan Lewis. - (EN) Monsieur le Président, je voudrais commencer par présenter mes excuses au Parlement pour être arrivé en retard ce matin. La raison en est simplement que l'on m'a fait part d'un horaire totalement différent. C'est pourquoi je pensais être arrivé à l'heure et je m'excuse donc sincèrement auprès des membres du Parlement pour ce malentendu. Si j'avais été au courant de l'horaire correct, il ne fait aucun doute que, par politesse envers le Parlement, j'aurais fait en sorte d'arriver à l'heure. Je m'excuse donc sincèrement pour ce malentendu. (Applaudissements) [...]

Je m'excuse encore une fois pour le malentendu qui a causé mon retard ce matin. (Applaudissements) (15 décembre 2005)

Ce discours est prononcé devant un public irrité par le retard du locuteur - plusieurs intervenants ont déjà fait des remarques ou ironisé sur son absence. Quand celuici arrive, il présente son retard comme causé par une erreur de communication, un programme incorrect qui lui aurait été remis. Le discours original en anglais le présente comme plus coupable que la traduction française : I was told there was an entirely different timescale vs on $m$ 'a fait part d'un horaire totalement différent. Il aurait été au moins naturel que le locuteur consulte l'horaire et ne se fie pas à une information qui lui aurait été simplement transmise. Du point de vue rhétorique, deux techniques sont à l'œuvre : d'une part, le locuteur sur lequel pèse l'opprobre public qualifie ce qui a provoqué son absence non pas de faute, d'incident malheureux, d'erreur, mais de malentendu, en utilisant un terme plus souple, plus élégant, délimitant de manière vague les responsabilités des deux parties, de telle sorte que l'absence, impardonnable, devient pour le public un simple retard, excusable dans certaines circonstances. D'autre part, le locuteur reprend la justification à plusieurs reprises, la triple répétition du terme malentendu dans l'extrait ci-dessus fonctionnant comme justification. A noter qu'avec la répétition du terme, l'acte de langage indirect de justification s'accomplit trois fois, avec des élaborations, et que, pour chaque emploi du terme, le locuteur accomplit un acte consistant à présenter ses excuses. Il est possible que la justification d'une erreur par un dysfonctionnement marginal de quelque système - un malentendu, apte à être dissipé, évacué, rectifié - soit plus efficace du point de vue rhétorique. On remarque bien que dans le cas exposé ci-dessus les applaudissements - signe du pardon public (ou bien de l'ironie des membres de la réunion ?) - suivent immédiatement les excuses.

Les contextes d'emploi révèlent que dans la conception du locuteur ordinaire, le malentendu est perçu comme le fruit d'un acte de communication mal mené, avec des conséquences négatives mais généralement réparables par une reformulation, une justification, une explication, une redéfinition, une élaboration concernant le sens des termes, ou bien un échange verbal. Des expressions telles que c'est un simple malentendu, ce n'est qu'un malentendu, c'est juste un malentendu montrent que les locuteurs considèrent qu'il y a des remèdes au désaccord. Toutefois, une situation qualifiée de malentendu peut rester au moins ambiguë si le destinataire du message n'accorde pas suffisamment de crédibilité au locuteur. Le communiqué de presse 
ci-dessous concerne le rapport rédigé par une commission temporaire du Parlement européen (2000-2001) chargée d'analyser les activités du réseau de veille stratégique mis en place par plusieurs Etats occidentaux (système d'interception Echelon) :

La commission temporaire sur le système d'interception Echelon entendait ce mardi 6 mars M. Lodewijk Briet, Directeur à la Commission européenne. Celui-ci entendait apporter des clarifications quant aux déclarations faites par M. Desmond Perkins, début février, devant la commission sur les relations avec la NSA (National Security Agency).

M. Briet a tout d'abord estimé que les malentendus étaient avant tout le résultat de formulations malheureuses. Il a toutefois voulu rassurer les députés sur les points les plus sensibles [...]

Ces déclarations ont cependant laissé perplexe un certain nombre de députés, d'aucuns allant même jusqu'à estimer que « les malentendus s'amplifient ». [...] En conclusion de cet échange de vues, le rapporteur, M. Gerhard Schmid (PSE, D) a estimé ne pas en savoir plus sur les raisons qui avaient amené M. Desmond Perkins à s'exprimer comme il l'avait fait le 5 février. (7 mars 2001) ${ }^{7}$

Cet extrait illustre, d'une part, une référence aux causes d'un (possible) malentendu, à savoir des « formulations malheureuses » et d'autre part, la situation au sein de laquelle ce qui pourrait être qualifié de malentendu par un locuteur prend des dimensions plus importantes dans la conscience des autres. L'extrait illustre aussi le besoin que ressent le locuteur, ou un tiers, d'apporter des clarifications concernant la portée sémantique et pragmatique des propos à l'origine du malentendu, de mettre en cause la façon de s'exprimer d'une autre personne, ou de se dire ignorant des raisons qui l'ont amené à s'exprimer d'une certaine manière. Par ailleurs, la notion de «malentendu interulturel» devient de plus en plus présente, de plus en plus invoquée comme le met en évidence l'extrait ci-dessous :

Question écrite posée par Nicole Thomas-Mauro (UEN) à la Commission

Objet : Management interculturel au sein de la Commission européenne

[...] A l'échelle de la Commission comme au niveau de la vie économique, une erreur d'analyse et d'appréciation ou tout simplement de compréhension peuvent [sic] avoir des conséquences graves (échec des projets, coût financier, coût en personnel $[\ldots])$.

La Commission a-t-elle déjà tenté de chiffrer le coût des malentendus interculturels ? [...] (7 novembre 2000) $)^{8}$

$7<$ http://www.europarl.europa.eu/sides/getDoc.do?type=PRESS\&reference=NR-20010307$1 \&$ format $=$ XML\&language $=\mathrm{FR}>$

8 Nicole Thomas-Mauro appartenait à l'UEN (Union pour l'Europe des Nations), un groupe politique national-conservateur eurosceptique du Parlement européen (actif dans l'intervalle 1999-2009). $<$ http://www.europarl.europa.eu/sides/getDoc.do?type $=$ WQ \& reference $=$ E-2000$3402 \&$ format $=$ XML\&language $=F R>$ 
On peut identifier ici un début de définition possible du terme de malentendu culturel, ou interculturel. Cette définition pourrait reprendre les idées répandues dans un large public : «Un malentendu culturel se produit quand quelque chose - un mot, un geste, un objet, un contexte social, à peu près tout ce qu'on peut imaginer - a des significations différentes dans deux cultures. » (ma trad., A.G.) ${ }^{9}$ Mais la littérature spécialisée permet de construire des définitions plus élaborées ( $c f$. Baré, 1985 ; Dewaele et Wourm, 2002 ; Rigo, 2004). Dans le cadre de cet article, le malentendu culturel pourrait se définir comme une erreur d'analyse, d'appréciation, de compréhension entre des personnes appartenant à des cultures différentes.

Dans la réponse donnée au sein de la Commission, on constate que dans les cas où le malentendu interculturel est invoqué, s'effectue un rapprochement notionnel entre le malentendu et d'autres conduites, pas nécessairement linguistiques, susceptibles d'entraver la communication et l'action dans l'environnement interculturel. Ce point est souligné dans l'intervention de M. Neil Kinnock, ancien membre de la Commission européenne et plus tard vice-président de celle-ci, en réponse à la question de Madame Thomas-Mauro, citée ci-dessus :

La Commission n'a pas entrepris d'études spécifiques sur ce sujet ni ne dispose d'une estimation des coûts susceptibles d'avoir été induits par les différences culturelles. Une estimation de ces coûts devrait bien entendu être fondée sur une analyse précise et fiable des causes et des conséquences des malentendus. La réalisation pratique d'une telle étude paraît cependant douteuse.

[...] Cette brochure de six pages souligne l'importance de la diversité culturelle et ses conséquences sur l'environnement de travail. Elle passe en revue les pièges (stéréotypes, malentendus, attitudes négatives, etc.) à éviter et donne des indications sur les comportements propres à améliorer la qualité de la relation interculturelle, lorsque cela est nécessaire. $\left(8\right.$ janvier 2001) ${ }^{10}$

Les justifications que l'on donne dans le contexte législatif pour faire des amendements aux projets de lois, peuvent constituer un autre exemple de « remède linguistique » à un malentendu:

La différence entre " brevet " et " brevet approprié " n'est pas évidente. Pour pallier tout malentendu, il convient d'énoncer la définition intégrale telle qu'elle figure à la directive 2001/25/CE au lieu de s'en tenir à une simple référence à cette directive. (2 décembre 2004) $)^{11}$

Ce commentaire relativement stéréotypé dans ce contexte montre que la définition d'un terme est un élément important pour prévenir ou dissiper un malentendu.

Dans La Nouvelle Rhétorique, Perelman et Olbrechts-Tyteca (1958) évoquent l'image du bâton à moitié plongé dans l'eau qui semble brisé à cause de la

$9<$ https://www.psychologytoday.com/blog/looking-in-the-cultural-mirror/201005/ cultural-misunderstandings $>$

$10<$ http://www.europarl.europa.eu/sides/getAllAnswers.do?reference=E-2000-3402\&language=FR $>$.

$11<$ http://www.europarl.europa.eu/sides/getDoc.do?type=REPORT\&reference=A6-2004$0057 \&$ format $=$ XML\&language $=\mathrm{FR}>$. 
réfraction - ce qui n'est qu'une illusion ${ }^{12}$. Ils mettent ainsi en opposition la réalité avec les apparences, un thème discuté de longue date dans la tradition philosophique. A partir de là, ils proposent le concept de dissociation, qu'ils considèrent comme une technique argumentative traitée plus récemment dans les cadres méthodologiques de la pragma-dialectique (van Rees, 2009).

Revenons sur l'exemple (6b) présenté dans la première section de l'article :

(6b) ... il y a, fatalement, à la base de tout amour passionné, un malentendu, une illusion généreuse, une erreur de jugement: une conception fausse qu'on s'est faite l'un de l'autre... (Martin du Gard, Les Thibault, L'Epilogue, 1940, p. 839) (TLF)

Le malentendu y est décrit comme ce que Perelman et Olbrechts-Tyteca (1958) appellent une dissociation. On dissocie une partie du contenu d'une notion afin de lui donner une nouvelle représentation. Dans ce cas, l'emploi de l'expression amour passionné permet la dissociation de la notion « amour » d'une nouvelle notion, celle d' « amour passionné », distincte de la notion originale " amour ». Ainsi, l'amour passionné repose, à la lumière de ce commentaire et de cette nouvelle définition, sur le malentendu, sur une illusion généreuse, une erreur de jugement, une conception fausse de l'autre. Les termes négatifs introduits dans cette définition persuasive ${ }^{13}$ (illusion, erreur, conception fausse) réinvestissent le contenu notionnel du terme malentendu de manière à dissocier cette forme ou type d'amour de ce qui est perçu comme « amour » dans la conception usuelle, commune du terme. Un tel commentaire vise à persuader que l'amour est distinct de l'amour passionné, qui reposerait, dans le contexte de cette représentation, sur une différence de représentation de la part des deux parties.

La dissociation se rapproche de la définition persuasive : le locuteur envisage une nouvelle définition d'une notion afin d'en valoriser certains aspects dans un but argumentatif. Il considère que cette redéfinition peut contribuer à l'acceptation par l'autre du point de vue qu'il avance explicitement ou implicitement. Dans l'extrait suivant, l'auteur prétend définir le terme socialiste, mais cette définition commence par l'élimination de certains aspects notionnels considérés comme nuisibles à

12 Voici ce que dit Jean-Jacques Rousseau à propos de cette illusion d'optique : « La première fois qu'un enfant voit un bâton à moitié plongé dans l'eau, il voit un bâton brisé : la sensation est vraie; et elle ne laisserait pas de l'être, quand même nous ne saurions point la raison de cette apparence. Si donc vous lui demandez ce qu'il voit, il dit : Un bâton brisé, et il dit vrai, car il est très sûr qu'il a la sensation d'un bâton brisé. Mais quand, trompé par son jugement, il va plus loin, et après avoir affirmé qu'il voit un bâton brisé, il affirme encore que ce qu'il voit est en effet un bâton brisé, alors il dit faux. Pourquoi cela ? Parce qu'alors il devient actif, et qu'il ne juge plus par inspection, mais par induction, en affirmant ce qu'il ne sent pas, savoir que le jugement qu'il reçoit par un sens serait confirmé par un autre. Puisque toutes nos erreurs viennent de nos jugements, il est clair que si nous n'avions jamais besoin de juger, nous n'aurions nul besoin d'apprendre : nous ne serions jamais dans le cas de nous tromper; nous serions plus heureux de notre ignorance que nous ne pouvons l'être de notre savoir. » (Rousseau, 1817 : 192-193.

13 La définition de dictionnaire est une définition lexicographique servant de terme de référence. La définition persuasive permet au locuteur d'employer le modèle syntaxique d'une définition de dictionnaire, en y introduisant des modifications ou des substitutions de contenu plus ou moins rapprochées de la signification du dictionnaire. Certains traits de la notion sont ainsi valorisés afin de l'utiliser dans un certain contexte avec le but de persuader. Un exemple de définition persuasive est : "Aimer c'est donner raison à l'être aimé qui a tort. " (Charles Péguy) $<$ http://dicocitations.lemonde.fr/citation-c-est-quoi-1-amour.php\#RxRDCmWpBAmW8KVh.99>. 
l'argumentation qui va suivre : «Le bon socialiste n'est ni celui qui rêve, ni celui qui renverse, ni celui qui violente. C'est celui qui cherche constamment le vrai progrès... » (Grünn, 1849 ; TLF) Les deux dissociations identifiées dans cet extrait, « le bon socialiste » et « le vrai progrès », indiquent au récepteur du message qu'il doit reconsidérer les notions de socialisme et de progrès dans une nouvelle optique, comme si les représentations de ces notions qu'il acceptait implicitement ne convenaient pas à la situation de communication donnée. Les points de départ de la discussion - les contenus notionnels implicitement disponibles et partagés par les participants à l'échange sont ainsi remis en question et redéfinis dans de nouveaux cadres. Ces dissociations peuvent être considérées comme des techniques argumentatives exploitant la fonction rhétorique de la définition et la définition comme figure. Dans l'extrait ci-dessus, les faits sont présentés implicitement comme si la résolution d'un malentendu exigeait le recours à une définition locale, valable seulement dans le contexte argumentatif donné, délimitant une nouvelle situation rhétorique. En introduisant une dissociation, l'auteur fait entendre qu'il y a une ambiguïté dans le discours précédent ou un désaccord entre les participants à l'échange. Son discours serait ainsi censé apporter une clarification. Il présente implicitement son intervention discursive comme une tentative de solution d'une différence d'opinion (réelle ou virtuelle) par le biais d'une discussion critique dont les points de départ sont remis en cause par la dissociation. La contribution métalinguistique de la dissociation est double, d'où son potentiel rhétorique : elle peut assurer le changement des points de départ de la discussion critique en faveur de l'intention argumentative, et elle semble résoudre un malentendu reposant sur des représentations notionnelles.

Cette approche repose sur une perspective argumentative maximale, propre à la pragma-dialectique. Dans des buts analytiques, cette discipline conçoit en effet tout échange linguistique comme argumentatif, dans le sens où le locuteur avance une thèse qu'il défend à travers son discours.

Dans cet extrait d'un communiqué de presse, le rapporteur rappelle les interventions des locutrices, qui avancent chacune (au moins) un point de vue défendu de manière implicite ou explicite :

Femmes israéliennes et palestiniennes : arrêter la spirale de la violence ${ }^{14}-$ $M^{\text {me }}$ Riad Dijani a témoigné de la difficulté des Palestiniens à vivre à Jérusalem en raison des contrôles incessants et des humiliations constantes, faisant part également d'une "perte de perspective d'avenir ». De culture arabe, mais de citoyenneté israélienne, $M^{\text {me }}$ Sibani a insisté sur les discriminations et la marginalisation croissantes dont sont victimes les Arabes en Israël. Selon elle,

14 La citation suivante contient la liste des participant(e)s à cet échange : « Le groupe de travail sur les droits de l'Homme du Parlement européen a procédé mardi, dans le cadre de la commission des affaires étrangères, à un échange de vues avec des femmes israéliennes et palestiniennes appartenant à différentes organisations féminines qui luttent pour une résolution pacifique du conflit au ProcheOrient. Il s'agissait de Mesdames Galia Golan (Fondatrice et dirigeante du mouvement La paix maintenant, Jérusalem Link et Bat Shalom), Debby Lerman (Coalition des Femmes pour la Paix), Suha Sibani (Directrice de l'Association arabe Commission pour l'Education) pour le côté israélien, de Mmes Janwa Riad Dijani (ministère palestinien de la Jeunesse à Jérusalem), Maha Abu Daye Shammas (Directrice du centre des femmes pour l'aide et le conseil juridique), et Samia Bamieh (Centre Jérusalem pour les femmes) du côté palestinien. »< <http://www.europarl.europa.eu/sides/getDoc. do?type $=$ PRESS\&reference $=$ NR-20020417-1\&format $=$ XML\&language $=F R>$. 
une intervention internationale est nécessaire pour préserver la dignité d'un peuple en proie au mépris et à la pauvreté. Pour $M^{\text {me }}$ Golan, représentant le courant de la gauche sioniste israélienne, il s'agit de remédier au malentendu répandu en Israël, selon lequel, à Camp David, Ehud Barak aurait offert beaucoup aux Palestiniens, qui y ont répondu par la violence. Or "la réalité dans les territoires occupés n'est pas connue de la plupart des Israéliens qui préfèrent se considérer comme des victimes ». Pourtant, nombreux sont ceux qui comprennent qu'une solution politique est nécessaire. L'Union européenne a un rôle fort à jouer dans la pacification du conflit afin de permettre un retour à la table des négociations. $\left(17\right.$ avril 2002) ${ }^{15}$

Prenons l'exemple de Mme Golan ; elle avance en même temps plusieurs points de vue :

1) Les Israéliens ont tort de se considérer comme des victimes.

2) Ils ont cette perception des faits parce qu'ils ne connaissent pas la réalité palestinienne.

3) La réalité est différente de celle qu'ils perçoivent - ils perçoivent une réalité distordue.

Dans l'extrait ci-dessus, elle opère une dissociation entre la situation réelle (la réalité dans les territoires occupés - accessible à ceux qui y vivent) et la représentation que s'en font la plupart des Israéliens, représentation fautive, issue d'un malentendu ou plutôt d'une méprise concernant les données réelles que l'on peut connaître sur place. Ceci revient à utiliser une autre dissociation implicite entre le statut réel des Israéliens et leur représentation en tant que victimes - représentation qui est locale, marquée subjectivement, et donc à rejeter tout en comprenant l'origine de la mécompréhension. L'explicitation et la dissociation jouent le rôle de remède au malentendu, mais aussi celui d'étape à franchir afin d'atteindre une solution. En même temps, présenter cette situation comme (issue d')un malentendu revient à l'évaluer comme moins dangereuse et, par conséquent, susceptible d'améliorations par des clarifications, des discussions, des débats entre les deux parties concernées.

\section{L'impossibilité de remédier au malentendu}

L'art contemporain trouve ses moyens d'expression dans les objets de la vie courante et s'expose parfois à être non seulement mal perçu et interprété, mais à susciter des « malentendus » qui peuvent conduire à l'anéantissement d'une œuvre.

La photo ci-dessous représente une installation intitulée Où allons-nous danser ce soir exposée au Museion de Bolzano en Italie ( $c f$. sitographie). Croyant qu'il s'agissait des restes d'une célébration organisée dans le musée, les employés du service de nettoyage ont fait place nette, envoyant tous les éléments de l'installation directement aux poubelles. 


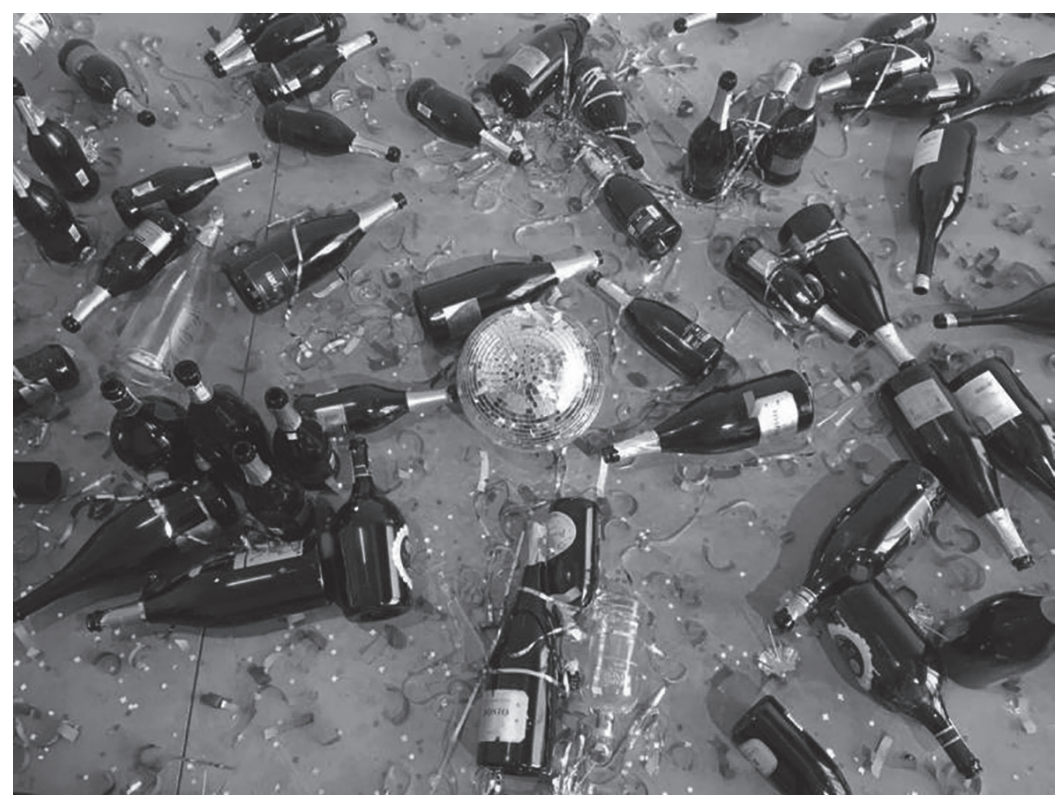

L'opera « Dove andiamo a ballare questa sera? » delle artiste Goldschmied and Chiari.

A la suite de cet incident, Charles Heimberg, professeur à l'Université de Genève, a posté un commentaire sur le réseau Twitter. Pour lui, l'intervention des employés résulte d'un malheureux malentendu :

En fait, il y a eu un malentendu, elle [l'installation] a été démantelée et la salle nettoyée par le personnel d'entretien... Elle sera bientôt réinstallée! (25 octobre $2015)^{16}$

Dans ce cas, on comprend qu'on a qualifié de malentendu une situation où il n'y a pas eu d'acte de langage. Cet incident naît d'une situation qui peut effectivement être regardée comme un malentendu. En effet, la situation décrite ci-dessus convient à la définition du $T L F$, où le malentendu est conçu comme une « divergence d'interprétation sur la signification de propos ou d'actes ». Le malentendu est ici le résultat de l'absence d'actes de langage qui auraient permis aux employés du service de nettoyage de concevoir ce qu'ils avaient sous les yeux comme une œuvre d'art. Dans cette situation, le malentendu était provoqué par la présence d'objets non conformes à l'usage habituel propre à l'endroit où ils se trouvaient. La construction et l'interprétation de la situation par les employés a été tout à fait naturelle : ce qu'ils voyaient était pour eux les restes d'une fête, ils étaient là pour nettoyer et ils ont nettoyé. Ils ne se sont pas posé de questions de nature esthétique, car ils n'étaient pas censés le faire. Je me souviens pour ma part de la remarque ironique d'un intellectuel roumain qui soulignait que certaines œuvres contemporaines sont parfois faites d'objets réels que les services de nettoyage

$16<$ http://www.repubblica.it/cronaca/2015/10/25/foto/bolzano_donne_delle_pulizie_gettano_opera_d_ arte_nella_spazzatura-125850377/1/?ref=HRESS-4\#1@heimbergch $>$. 
peuvent démonter et mettre à la poubelle à la fin de l'exposition. L'œuvre d'art existe comme telle de par le statut qui lui est donné par l'exposition (temporaire) dans un musée et elle cesse de l'être à la fin de l'événement. Jamais une telle œuvre ne sera remise dans le même état ou reconstituée. L'acte artistique, dans un tel cas, consiste à organiser et disposer des objets ordinaires en leur enlevant leur caractère quotidien ; il leur permet de sortir du commun et de l'anonymat et leur donne accès au statut supérieur d'œuvre d'art. Le visiteur du musée se trouve confronté à des objets qui ne peuvent pas prétendre au statut de composantes d'une œuvre d'art. Pourtant, l'interprétation du visiteur se fait dans le cadre conventionnel dans lequel le plonge sa visite : la plupart des objets placés dans les salles doivent être considérés comme, ou au moins interprétés comme, des œuvres d'art. Par conséquent, il va accepter plus ou moins cette confrontation. Or, l'œuvre dont il est question ici a plutôt le statut d'une collection d'objets. Les employés du service de nettoyage effectuent un acte qu'en fin d'exposition le personnel du musée fera aussi - peut-être avec plus de précautions et en respectant un règlement quelconque. Par ailleurs, pour le musée, il n'y a aucun intérêt à garder cette œuvre de circonstance ou à la restituer à l'artiste. Celui-ci pourra la reconstituer. La leçon à tirer de cet incident ne manque pas d'intérêt : l'installation artistique en question prend sa signification d'œuvre d'art à l'intérieur de l'espace muséal - sa signification est artistique et indiquée comme telle par le biais d'un acte de parole conventionnel : l'inscription d'un titre et du nom des auteurs sur le cartel qui l'accompagne. Dans le cas discuté, l'œuvre constituée d'une collection d'objets quotidiens risque d'être interprétée pour ce qu'elle est, un assemblage d'objets ordinaires, et de ne pas bénéficier de l'interprétation bienveillante du consommateur d'art contemporain. Les employés du service de nettoyage construisent leur interprétation sur la base des données disponibles - car ils ne sont pas censés lire les cartels : ils constatent la présence d'objets ordinaires en un endroit où ils ne devraient pas se trouver - «C'est donc sale », on nettoie, on débarrasse l'endroit de tout ce qui n'est pas à sa place.

Charles Heimberg explique le geste en termes de malentendu, mais en est-ce vraiment un ? Le schéma ci-dessous propose une représentation séquentielle des étapes qu'auront traversées les employés du service de nettoyage.

\begin{tabular}{|c|c|c|c|c|c|c|}
\hline $\begin{array}{l}\text { Perception de l'objet } \\
\text { absence d'acte de parole } \\
\text { justificatif de la présence } \\
\text { de l'objet }\end{array}$ & $\rightarrow$ & $\begin{array}{l}\text { Interprétation } \\
\text { individuelle/de } \\
\text { groupe }\end{array}$ & $\rightarrow$ & $\begin{array}{l}\text { Raisonnement explicatif = } \\
\text { justification personnelle } \\
\text { concernant la présence de } \\
\text { l'objet }\end{array}$ & $\rightarrow$ & Action \\
\hline & & $\begin{array}{c}\text { interprétation } \\
\text { erronnée }\end{array}$ & & Malentendu & & \\
\hline
\end{tabular}

Le malentendu dont parle l'auteur du tweet repose moins cette fois-ci sur une divergence d'interprétation que sur l'interprétation fâcheuse d'un acte de signification. La signification des objets était artistique, mais elle n'a pas été reconnue comme telle. Les objets ont été pris pour ce qu'ils étaient, et non comme composantes d'une œuvre d'art. Paradoxalement, l'artiste avait joué sur un quiproquo, qui n'a pas été identifié comme tel par les employés. 
Le malentendu ne conduit pas ici à un désaccord, mais à une action irréversible, comme c'est le cas d'un grand nombre de malentendus auxquels on ne peut guère remédier.

\section{Remarques finales}

L'approche choisie pour cet article est fondée sur une perspective linguistique et laisse par conséquent de côté un grand nombre de réflexions portant sur le malentendu dans des études comportementales, interculturelles, sociales et philosophiques. ${ }^{17} \mathrm{~A}$ partir des définitions lexicographiques de la notion, l'article traite principalement de ce que les locuteurs ordinaires qualifient de malentendu et de l'exploitation persuasive de la notion : comment s'excuser, demander des précisions, des élaborations du discours antérieurs à partir de la qualification d'une situation comme « malentendu ». Les locuteurs qualifient de « malentendu » ou considèrent comme telle une interprétation involontaire incorrecte ou fautive d'une action, d'un événement, de certains énoncés ou paroles. L'acte d'interprétation peut appartenir à un interactant auquel était destiné l'acte discursif ou non discursif à l'origine de la situation qui lui était apparue comme un malentendu. La situation ainsi représentée peut être assez complexe et difficile à reconstruire. L'acte initial, à l'origine de la situation peut être mal mené, incorrect, ambigu, fautif, défectueux en quelque sorte, tout comme peut l'être l'acte d'interprétation. Les conséquences du malentendu peuvent être plus ou moins graves, réparables ou irréparables. Quand elles ne sont pas très graves, se représenter la situation comme un malentendu et/ou la qualifier comme telle engage les responsables présumés sur la voie des excuses, des justifications, des reformulations, des explications, des précisions concernant les causes qui l'ont engendrée et les ressorts à faire jouer afin d'y remédier. Si les conséquences sont plus graves, voire irréparables, les excuses et les actes de justification ou de reformulation ne sont plus acceptables; ils n'ont plus d'objet, car le remède n'est pas disponible. Toutefois, représenter une situation comme un malentendu est un mouvement rhétorique et stratégique destiné à diminuer la culpabilité ou, au moins, la responsabilité de l'un ou de plusieurs interactants.

La qualification d'une situation de malentendu se fait de manière explicite, par l'usage de ce terme. Une situation est présentée de manière implicite comme un malentendu par l'emploi d'un large éventail d'expressions dont le recensement et l'inventaire non exhaustif pourrait ouvrir des pistes de recherche en linguistique, en rhétorique et dans la théorie de l'interaction. Etablir un corpus de travail adéquat serait une étape préliminaire nécessaire. L'exploitation des sténogrammes des réunions des organismes européens, utilisés ici comme base de travail, me semble constituer un bon point de départ. La qualification d'une situation discursive comme malentendu revient pour le locuteur qui s'en charge à effectuer un acte de langage de nature déclarative ; il s'agit d'une déclaration d'usage dans le sens où l'énoncé ou l'interprétation à l'origine du malentendu interlocutif est déclaré(e) incorrecte, ce qui appelle une reformulation.

17 Je remercie particulièrement Françoise Lauwaert et Laurent Legrain de leur patience immense, de leurs efforts de parcourir la première version inachevée de mon étude, et surtout pour toutes leurs suggestions et remarques. Je remercie aussi les deux relecteurs anonymes pour leurs suggestions et propositions de révision. 
C'est dans cette perspective que j'ai proposé une typologie " primaire ", de départ, du malentendu. C'est un outil dont on pourrait se servir ultérieurement dans les cadres disciplinaires plus étroits de la théorie des actes de langage, de la rhétorique et de la pragma-dialectique, pour des analyses plus poussées.

\section{Références citées}

*** [DAF] Dictionnaire de l'Académie françoise (1814), revu, corrigé et augmenté par l'Académie ellemême, $5^{\mathrm{e}}$ édition, tome 2. Paris : Bossange et Masson, Garnery, Henri Nicolle.

*** [TLF] Trésor de la langue française, édition informatisée, $<\mathrm{http}: / / \mathrm{www} . \mathrm{cnrtl} . \mathrm{fr} />$.

Austin, John L., 1962. How to do things with words (The William James lectures, Harvard University, 1955 , série de conferences). Oxford : Oxford University Press/Clarendon Press.

BARÉ, Jean-François, 1985. Le malentendu Pacifique. Paris : Hachette.

Darmesteter, Arsène et Adolphe HatzFeld, 1890. Dictionnaire général de la langue française du commencement du $17^{e}$ siècle jusqu'à nos jours, précédé d'un traité de la formation de la langue, 2 tomes; t. $1:$ A - F/, t. 2 : G-Z, 5 édition.

DASCAL, Marcelo, 1999. « Introduction : Some questions about misunderstanding », Journal of Pragmatics, 31, pp. 753-762.

Dewaele, Jean-Marc et Nathalie Wourm, 2002. « L'acquisition de la compétence sociopragmatique en langue étrangère ", Revue française de linguistique appliquée, 2 (Vol. VII), pp. 139-153. <www.cairn.info/ revue-francaise-de-linguistique-appliquee-2002-2-page-139.html>.

Fraser, Bruce, 1993. « No conversation without misrepresentation », in Herman Parret (éd.), Pretending to communicate, pp. 143-153. Berlin : de Gruyter.

Kellerer, Sidonie, Astrid Nierhoff-Fassbender, Alice Perri-Marsol, Fabien Théofilakis (éds), 2008. « Malentendu/Missverständnis. Kultur zwischen Kommunikation und Störung ». Forum, Vol. 4. Würzburg : Königshausen and Neumann.

LAFAYE, Pierre Benjamin, 1837. "Erreur », Encyclopédie des gens du monde ; répertoire universel des sciences, des lettres et des arts; avec des notices sur les principales familles historiques et sur les personnages célèbres, morts et vivants, par une société de savants, de littérateurs et d'artistes, français et étrangers, Tome neuvième. Paris : Librairie de Treuttel et Würtz.

LaVEaux, Jean-Charles, 1820 (1828). Nouveau dictionnaire de la langue française, $2^{\mathrm{e}}$ édition, revue, corrigée et augmentée, tome second. Paris : Deterville et Ledentu.

-, 1826. Dictionnaire synonymique de la langue française. Paris : Alexis Eymery.

Mortensen, C. David, 2006. Human conflict : Disagreement, misunderstanding, and problematic talk. New York/Oxford : Rowman and Littlefield.

Perelman, Chaïm et Lucie Olbrechts-Tyteca, 1958. La nouvelle Rhétorique. Traité de l'Argumentation, deux volumes, Collection « Logos ». Paris : Presses Universitaires de France.

ReEs, Agnes van, 2009. Dissociation in argumentative discussions. A pragma-dialectical perspective, Argumentation Library. Amsterdam : Springer.

Rigo, Bernard, 2004. Lieux-dits d'un malentendu culturel, nouvelle éd. revue et augmentée. Papeete : Au Vent des îles.

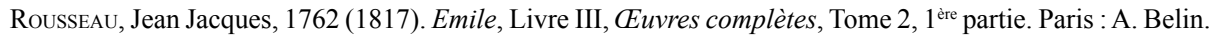

SeArle, John R., 1969 (1972). Speech acts. Cambridge : Cambridge University Press. (Les actes de langage. Essai de philosophie linguistique, Collection « Savoir », Préface d'Oswald Ducrot, traduction par Hélène Pauchard. Paris : Hermann). 
-, 1979 (1982). Expression and meaning. Cambridge : Cambridge University Press. (1982. Sens et expression. Etudes de théorie des actes de langage, traduction et préface par Joëlle Proust. Paris : Editions de Minuit). 